\title{
Orbital Kondo spectroscopy in a double quantum dot system
}

\author{
L. Tosi, ${ }^{1}$ P. Roura-Bas, ${ }^{2}$ and A. A. Aligia ${ }^{1}$ \\ ${ }^{1}$ Centro Atómico Bariloche and Instituto Balseiro, Comisión Nacional de Energía Atómica, 8400 Bariloche, Argentina \\ ${ }^{2}$ Departamento de Física, Centro Atómico Constituyentes, Comisión Nacional de Energía Atómica, Buenos Aires, Argentina
}

(Received 9 September 2013; published 20 December 2013)

\begin{abstract}
We calculate the nonequilibrium conductance of a system of two capacitively coupled quantum dots, each one connected to its own pair of conducting leads. The system has been used recently to perform pseudospin spectroscopy by controlling independently the voltages of the four leads. The pseudospin is defined by the orbital occupation of one or the other dot. Starting from the SU(4) symmetric point of spin and pseudospin degeneracy in the Kondo regime, for an odd number of electrons in the system, we show how the conductance through each dot varies as the symmetry is reduced to SU(2) by a pseudo-Zeeman splitting, and as bias voltages are applied to any of the dots. We analyze the expected behavior of the system in general, and predict characteristic fingerprint features of the $\mathrm{SU}(4) \rightarrow \mathrm{SU}(2)$ crossover that have not been observed so far.
\end{abstract}

DOI: 10.1103/PhysRevB.88.235427

PACS number(s): 73.63.Kv, 72.15.Qm

The Kondo effect, originally observed in systems of magnetic impurities in metals, ${ }^{1}$ has reappeared more recently in the context of semiconductor quantum-dot (QD) systems, with a single "impurity," in which an unprecedented control of the parameters could be achieved..$^{2-5}$ The effect is characterized by the emergence of a many-body singlet ground state formed by the impurity spin and the conduction electrons in the Fermi sea.

The role of the impurity spin can be replaced by another quantum degree of freedom (pseudospin) that distinguishes degenerate states, such as orbital momentum. A particularly interesting case is when both a twofold orbital degeneracy and spin degeneracy are present, leading to an SU(4) Kondo effect. ${ }^{6-19}$ This exotic Kondo effect has been observed in different systems, such as quantum dots in carbon nanotubes, ${ }^{9-15}$ silicon nanowires, ${ }^{16}$ and organic molecules deposited on $\mathrm{Au}(111) .^{18}$

Recently, a double QD with strong interdot capacitive coupling, and each QD tunnel coupled to its own pair of leads, has been experimentally ${ }^{19,20}$ and theoretically ${ }^{19,21-23}$ studied [see Fig. 1(a)]. The occupation of one QD or the other plays the role of the pseudospin. These occupations, the tunneling matrix elements, and the voltages at the four leads can be controlled independently. While the spin degeneracy can be broken by a magnetic field, this also affects the conduction leads. Instead, a pseudo-Zeeman splitting can be applied on the QDs solely, opening the exciting possibility to explore in detail the orbital structure of the SU(4) Kondo state and how it is changed as the pseudo-Zeeman field reduces the symmetry to $\mathrm{SU}(2)$.

So far, the theoretical study of this system has been concentrated in equilibrium properties, for which accurate techniques such as numerical renormalization group (NRG) and density-matrix renormalization group can be applied. A much richer physics is expected in the nonequilibrium situation, which arises for finite bias voltages between the leads connected to any of the QDs in the experiment, because of the presence of inelastic processes. Unfortunately, the theoretical treatment is much more difficult in this case. For one QD, the experimental study at finite bias voltages ${ }^{24}$ allowed the test of universality and scaling relations within different nonequilibrium theories. ${ }^{25,26}$ Here we use the Keldysh formalism within the noncrossing approximation (NCA), ${ }^{27,28}$ which reproduces well the scaling relations mentioned above ${ }^{29}$ and was also successfully used to interpret experimental results on a controlled crossover between SU(4) and SU(2) Kondo states driven by magnetic field in a nanoscale Si transistor, ${ }^{16}$ and quantum phase transitions involving singlet and triplet states. ${ }^{30}$

We report calculations of the conductances through both QDs in the general nonequilibrium case. We describe in particular nontrivial changes in the conductance through one QD as a voltage is applied to the other. We also describe how the spectral densities evolve under application of different bias voltages. Fingerprints of the $\mathrm{SU}(4) \rightarrow \mathrm{SU}(2)$ crossover are predicted.

Our starting model is the SU(4) Anderson model which mixes a singlet configuration $|s\rangle$ with two degenerate spin doublets $|i \sigma\rangle(i=1$ or 2$)$ corresponding to one additional electron (or hole) in QD $i$, through couplings $\Gamma_{1}=\Gamma_{2}$ to a continuum of extended states. The Hamiltonian is

$$
\begin{aligned}
H= & E_{s}|s\rangle\left\langle s\left|+\sum_{i \sigma} E_{i}\right| i \sigma\right\rangle\langle i \sigma|+\sum_{i v k \sigma} \epsilon_{v k} c_{v k i \sigma}^{\dagger} c_{v k i \sigma} \\
& +\sum_{i v k \sigma}\left(V_{i}^{v}|i \sigma\rangle\langle s| c_{v k i \sigma}+\text { H.c. }\right),
\end{aligned}
$$

where $c_{v k i \sigma}^{\dagger}$ create conduction states at the source $(v=S)$ or drain $(v=D)$ lead, and $V_{i}^{v}$ is the hopping between the lead $v$ and dot $i$, assumed independent of $k$ for simplicity. The symmetry is reduced to $S U(2)$ by a pseudo-Zeeman splitting $\delta=E_{2}-E_{1}$, which raises the energy of a particle in QD2 $\left(E_{2}\right)$ with respect to the corresponding one for QD1 $\left(E_{1}\right)$. The tunnel couplings of each QD to the leads are $\Gamma_{v i}=2 \pi \sum_{k}\left|V_{i}^{v}\right|^{2} \delta\left(\omega-\epsilon_{v k}\right)$, and we take the unit of energy $\Gamma_{i}=\Gamma_{S i}+\Gamma_{D i}=1$ unless otherwise stated. $\Gamma_{i}$ correspond to the total width at half maximum of the spectral density in the noninteracting system. It is of the order of $20 \mu \mathrm{eV}$ in the experiments. ${ }^{19,20}$ Since charge configurations with two particles are excluded, the model assumes infinite on-site repulsions $U_{i}$ and interdot repulsion $U_{12}$. This assumption is not essential in the Kondo regime for one particle (electron or hole) in the system, which is the focus of our study. A scheme of the setup and basic parameters is represented in Fig. 1. In real systems, $\Gamma_{1} \neq \Gamma_{2}$ and SU(4) symmetry is lost 

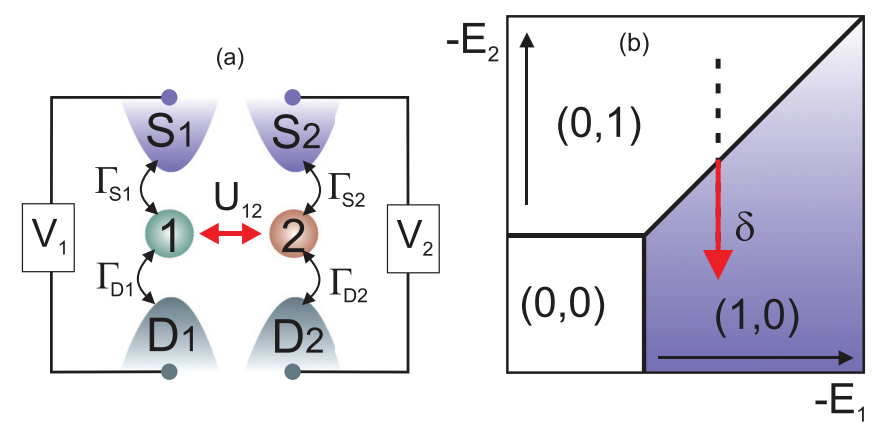

(c)
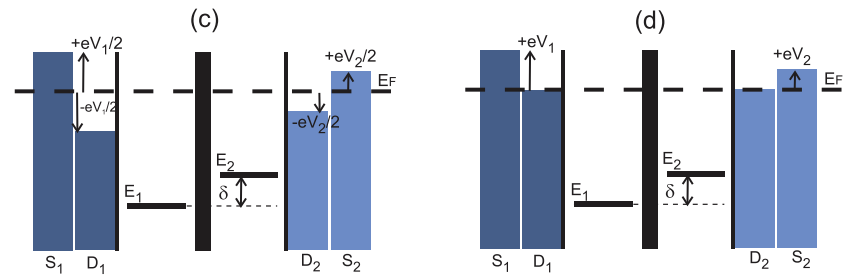

FIG. 1. (Color online) (a) Scheme of the experimental setup (Refs. 19 and 20). (b) On-site energies of our model and occupations of both quantum dots. Our convention for application of voltages is in (c) for a symmetric voltage drop and (d) for voltage applied only to the source leads.

even for $\delta=0$. However, we find that tuning appropriately $\delta$, the equilibrium spectral densities for both dots $\rho_{i}(\omega)$ can be made to coincide at low temperatures. This indicates that the SU(4) symmetry is recovered as an emergent (approximate) symmetry ${ }^{31}$ at low temperatures.

The NCA formalism is explained in Ref. 32 (for a more general case) and in the Supplemental Material. ${ }^{33}$ We start reporting the differential conductances $G_{i}=d I_{i} / d V_{i}$ in the SU(4) symmetric case. In Fig. 2 we show $G_{2}$ as a function of both $V_{i}$ for symmetric voltage drops $\left[V_{S i}=-V_{D i}=V_{i} / 2\right.$; see Fig. 1(c)] and coupling to the leads $\left(\Gamma_{S i}=\Gamma_{D i}\right)$. By SU(4) symmetry, $G_{1}$ has the same form interchanging $V_{1}$ and $V_{2}$. For $V_{1}=V_{2}=0$, there is a maximum of the conductance due to the SU(4) Kondo effect. ${ }^{10,11,17}$ At temperature $T=0$, this maximum is slightly below $G_{0} / 2$, where $G_{0}=2 e^{2} / h$, due to

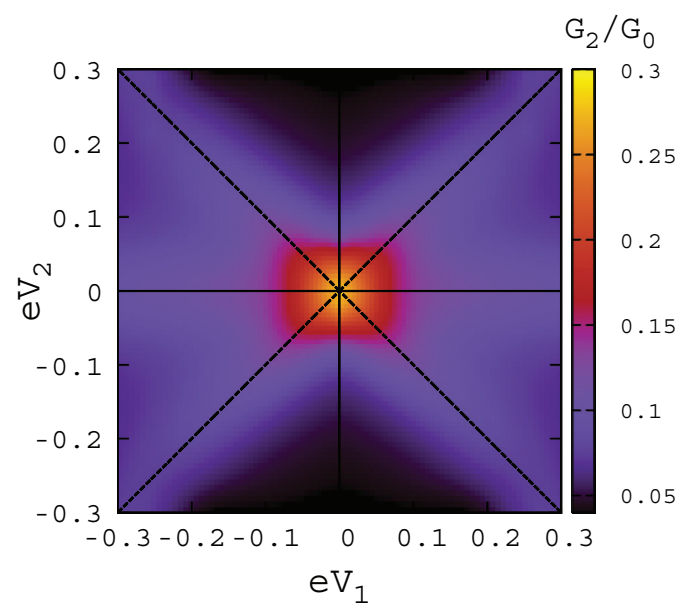

FIG. 2. (Color online) Conductance of QD2 as a function of $V_{1}$ and $V_{2}$ for $\delta=0, E_{1}=-4$, and $T=0.005$. some degree of intermediate valence, according to the Friedel sum rule $\mathrm{e}^{14,17,33}$ (the filling is slightly below $1 / 4$ ). Application of either $V_{1}$ or $V_{2}$ tends to destroy the Kondo effect and the conductance decreases. Note that application of $V_{2}$ has a stronger effect on decreasing $G_{2}$ than $V_{1}$. In fact for $V_{2}=0$ and any $V_{1}$, one expects that the spin Kondo effect on QD2 still remains, although weakened, and this is consistent with our results. As in the usual $\mathrm{SU}(2)$ Kondo effect, $G_{i}\left(V_{i}\right)$ drops to $G_{i}(0) / 2$ at a bias voltage such that $e V_{i} \approx T_{K}^{\mathrm{SU}(4)}$, where $T_{K}^{\mathrm{SU}(4)}$ is the Kondo temperature for $\delta=0$ as discussed below. For our parameters, $T_{K}^{\mathrm{SU}(4)} \approx 0.02$ and it increases to near 0.3 if $E_{1}$ is changed from -4 to -2 . Since experimentally temperatures $T \approx 0.1$ can be reached, and $E_{1}$ can be tuned, a wide range of ratios $T / T_{K}^{\mathrm{SU}(4)}$ is accessible.

Nontrivial correlation effects between both QDs are apparent in the fact that $G_{i}$ increases on the lines $V_{1}= \pm V_{2}$. This is related to the evolution of the spectral densities $\rho_{i}(\omega)$ as both $V_{i}$ are varied. We find that keeping $V_{2}=0$ and increasing $V_{1}$ (or conversely) the Kondo peak at $\omega=0$ in $\rho_{i}(\omega)$ is weakened and two peaks at $\omega \approx \pm e V_{1} / 2$ split from it. In the general case, when both $V_{i} \neq 0$, four peaks are present in both $\rho_{i}(\omega)$ for $\omega \approx \pm e V_{i} / 2$. When $V_{1}= \pm V_{2}$ these peaks merge in two more intense peaks and therefore an increase in both $G_{i}$ is expected.

In Fig. 3 we show how the $G_{i}$ change when a finite pseudo-Zeeman splitting $\delta$ is introduced. It is known that the spectral density of the dot with lower energy $\rho_{1}(\omega)$ has still the Kondo peak near $\omega=0$ and an additional peak for $\omega \approx-\delta$, while $\rho_{2}(\omega)$ has only a peak for $\omega \approx \delta$ (see Fig. 4). ${ }^{10,14}$ As a consequence, only $G_{1}$ has a peak near $V_{1}=V_{2}=0$, while $G_{2}$ is vanishingly small at that point. The energy scale of the variation of $G_{1}$ with $V_{1}$ is again given by the Kondo temperature $T_{K}$, but it is smaller than that of the $\mathrm{SU}(4)$ case.

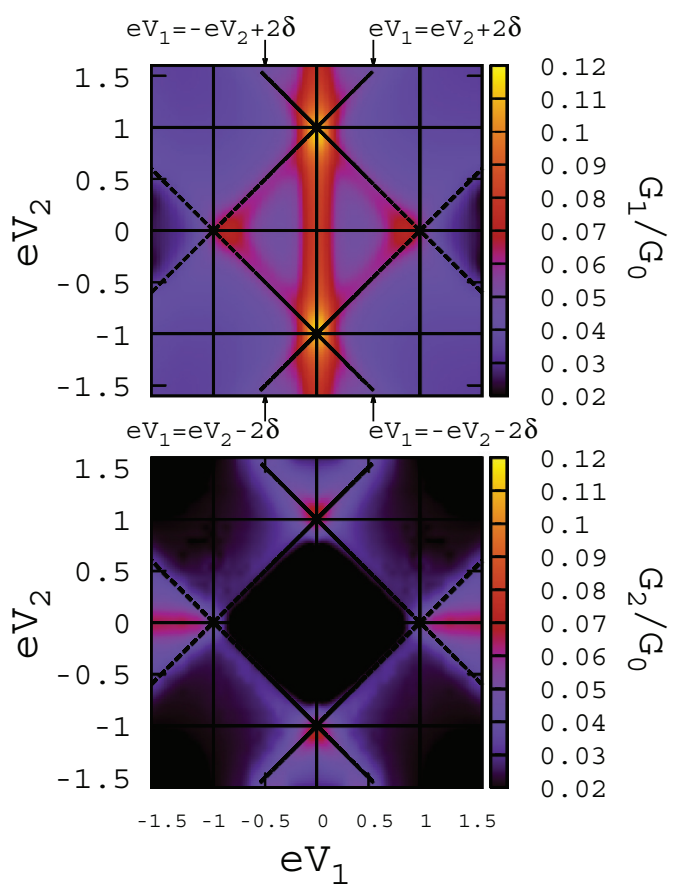

FIG. 3. (Color online) Conductance of (a) QD1 and (b) QD2 as a function of $V_{1}$ and $V_{2}$ for $\delta=E_{2}-E_{1}=0.5, E_{1}=-4$, and $T=0.005$. 

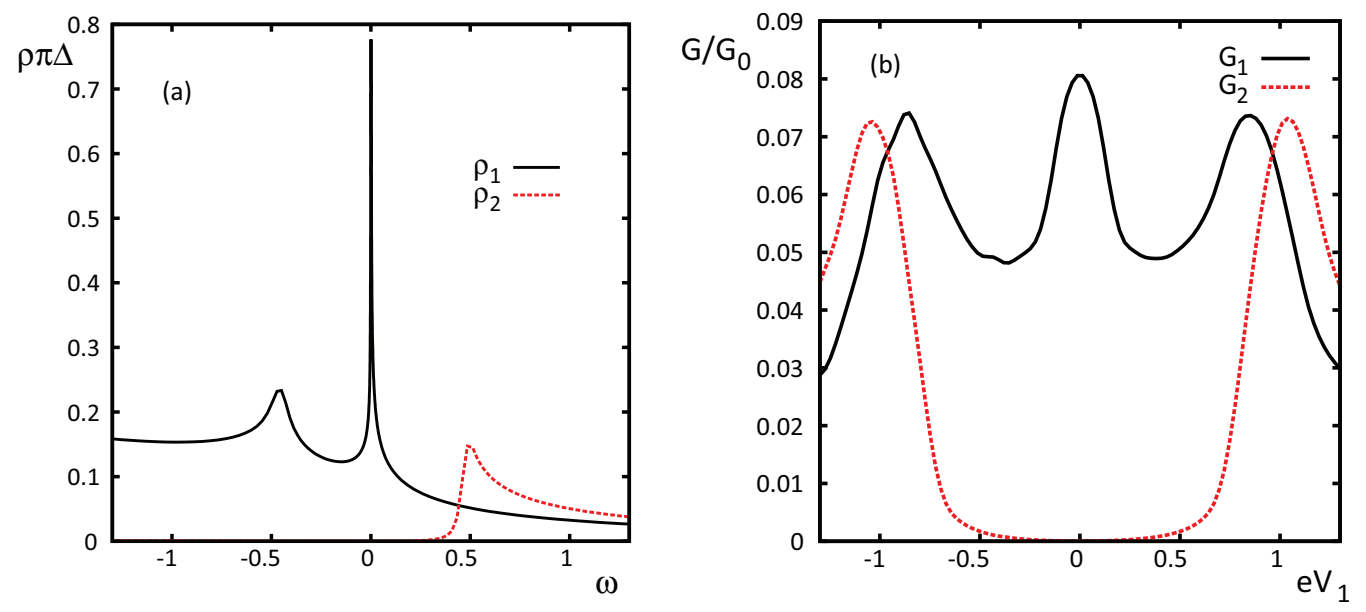

FIG. 4. (Color online) (a) Spectral densities for QD1 (black solid line) and QD2 (red dashed line). (b) $G_{i}$ as a function of its bias voltage $V_{i}$ keeping the other bias voltage 0 . Parameters as in Fig. 3. $\Delta=\Gamma / 2$.

We have found that the binding energy of the singlet ground state obtained from a simple variational calculation can be described by the following expression: ${ }^{14}$

$$
\begin{aligned}
\frac{T_{K}(\delta)}{T_{K}(0)} & =\sqrt{1+\tilde{\delta} / d+\tilde{\delta}^{2}}-\tilde{\delta}, \\
\tilde{\delta} & =\frac{\delta}{2 T_{K}(0)}, \quad d=\frac{D}{2 T_{K}(0)},
\end{aligned}
$$

where $T_{K}(0)=T_{K}^{\mathrm{SU}(4)}$ with $T_{K}^{\mathrm{SU}(N)} \approx D \exp \left[2 \pi E_{1} /(N \Gamma)\right]$ and $D$ is half the bandwidth (we took $D=10$ ). Note that $T_{K}(\infty)=T_{K}^{\mathrm{SU}(2)}$, so that Eq. (2) interpolates between the known Kondo temperature for both limits. ${ }^{14}$ The width of the Kondo peak calculated within the NCA agrees remarkably well with this expression. ${ }^{14}$ Specifically for $E_{1}=-4$ the total width of the Kondo peak in the spectral density is found to be $1.2 T_{K}(\delta)$.

We find that for $T<T_{K}(\delta)$ and $V_{1}>T_{K}(\delta), G_{1}\left(V_{1}\right)$ for $V_{2}=0$ presents a structure with three peaks [see Fig. 4(b)] which has not been observed experimentally yet and is characteristic of the $\mathrm{SU}(4) \rightarrow \mathrm{SU}(2)$ crossover. ${ }^{38}$ Since $T_{K}(\delta)$ varies over several orders of magnitude, we believe that an experimental observation of this fingerprint of the crossover is near the present experimental possibilities. ${ }^{19,20}$

Another apparent feature is the increase of both conductances along the lines $e V_{1}= \pm e V_{2} \pm 2 \delta$ (shown dashed in Fig. 3). This can be understood from the onset of cotunneling events near these equalities. ${ }^{34}$ Let us assume first that $V_{1}=$ $V_{2}=0$. The cotunneling event in which the electron that occupies QD1 jumps to its source (S1) or drain (D1) lead and an electron from S2 or D2 jumps to QD2 is inhibited because of the energy cost $\delta$. However, when $e\left|V_{2}\right|$ reaches $2 \delta$, an event of this type becomes possible, in which as a net result an electron flows from S2 to D2 or conversely depending on the sign of $V_{2}$, and another electron moves from QD1 to QD2 with a possible spin flip. In a second event the electron of QD2 jumps to its lead of less chemical potential and an electron from S1 or D1 jumps to QD1, leaving the QDs in the same charge configuration as initially. This results in an increase of the current flow $I_{2}$ and thus to a peak in the conductance $G_{2}$.
On average $I_{1}=0$. However, a small $\left|V_{1}\right|$ breaks the symmetry between $\mathrm{S} 1$ and D1 in the above events, leading to a large $G_{1}$ also. A similar reasoning can be followed for nonzero $V_{1}$.

More insight into the structure of the nonequilibrium conductance is obtained from the spectral densities $\rho_{i}(\omega)$. At equilibrium and low temperatures, both $\rho_{i}(\omega)$ have a Kondo peak slightly above the Fermi energy (which we take as the origin of energies) for $\delta<T_{K}^{\mathrm{SU}(4)}{ }^{14}$ while for $\delta>T_{K}^{\mathrm{SU}(4)}$, as seen in Fig. 4(a), the Kondo peak in $\rho_{1}(\omega)$ moves to the Fermi energy and an inelastic peak near $-\delta$ appears $(\delta=0.5$ in the figure). The width of the Kondo peak is $\sim T_{K}(\delta)$. Instead $\rho_{2}(\omega)$ has only an inelastic peak near energy $\delta$. We find that the width of both inelastic peaks is of the order of $T_{K}^{\mathrm{SU}(4)}$ for small $\delta$ (but $\delta>T_{K}^{\mathrm{SU}(4)}$ in order to ensure that the inelastic peak is split from the Kondo peak) and increases with increasing $\delta$. This behavior is reminiscent of the evolution of the peaks of the ordinary SU(2) Kondo model under an applied magnetic field, which has been studied by Bethe ansatz techniques. ${ }^{35}$

The equilibrium spectral densities can be investigated by orbital spectroscopy controlling the parameters so that the configuration is similar to that used in scanning tunneling spectroscopy (STS). Specifically if $\Gamma_{S i} \gg \Gamma_{D i}$, and only the potential at one of the drains $V_{D i}$ is displaced from the Fermi level, then the dots are in equilibrium with the source leads and for $T \ll T_{K}(\delta), G_{i} \propto \rho_{i}\left(e V_{i}\right)$. Our calculations show that a ratio $\Gamma_{S i} / \Gamma_{D i}=9$ is enough to reach this STS regime. This property was used to study the equilibrium spectral density and to compare it with that resulting from an NRG calculation for a case in which both spin and pseudospin Zeeman terms were present. ${ }^{19}$ However, this destroys the Kondo effect and the two-peak structure like that shown in Fig. 4(a) remains unexplored. In Fig. 5 we show the evolution of the spectral density starting from the SU(4) case and increasing $\delta$ for parameters reached experimentally in recent work, ${ }^{19,20}$ in particular $\Gamma_{i}=20 \mu \mathrm{eV}, T=23 \mathrm{mK}$. While the peaks become sharper at lower temperature, the displacement of the Kondo peak to the Fermi energy from above, and the splitting of the inelastic peak as $\delta$ increases, can be clearly seen. Due to the limitations of resolution of NRG at finite energies ${ }^{36,37}$ our NCA results are a useful complement at equilibrium ${ }^{42}$ and have 


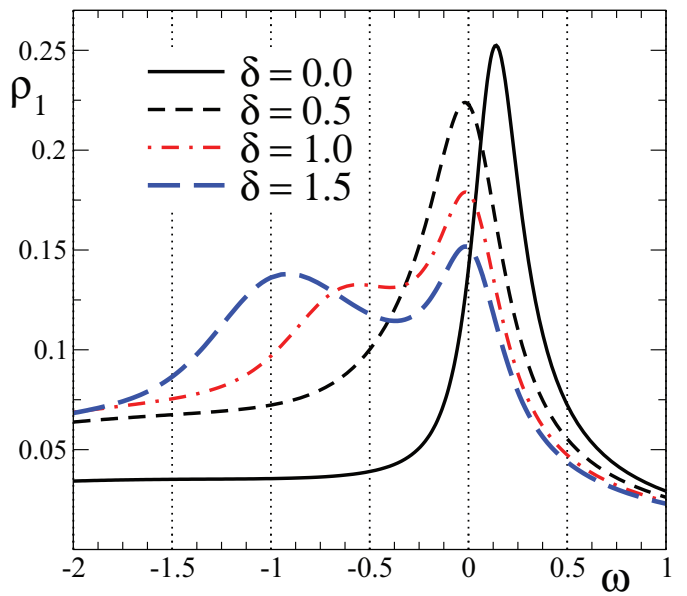

FIG. 5. (Color online) Spectral density for QD1 for $E_{1}=-2.5$, $T=0.1$, and several values of $\delta$.

the advantage that they can be extended to the nonequilibrium situation.

In general, and particularly for a symmetric voltage drop, the spectral densities change under application of bias voltages $V_{i}$. Assuming as a first crude approximation that the $\rho_{i}(\omega)$ are fixed, one expects that $G_{1}\left(V_{1}\right)$ has a peak at $V_{1}=0$ corresponding to the Kondo peak in $\rho_{1}(\omega)$, and two peaks at $V_{1}= \pm 2 \delta / e$ corresponding to the inelastic peak of $\rho_{1}(\omega)$. This is in fact what happens for $V_{2}=0$ [see Fig. 4(b)] but not for $V_{2} \neq 0$ [see Fig. 3(a)]. Similarly one expects only inelastic peaks at $V_{2}= \pm 2 \delta / e$ for $G_{2}\left(V_{2}\right)$, as it happens for $V_{1}=0$ but not for $V_{1} \neq 0$.

The differences with the expected behavior for rigid bands when both $V_{i} \neq 0$ are due to changes in the spectral weight with respect to the equilibrium case. To illustrate these changes we consider the nonequilibrium situation represented in Fig. 4(d) of Ref. 20, in which the coupling to the source leads is larger and the voltages are applied only in one of these sources $V_{S i}$, keeping the other three voltages at zero. Specifically we keep $\Gamma_{i}=1$ but use $\Gamma_{S 1} / \Gamma_{D 1}=3$ and $\Gamma_{S 2} / \Gamma_{D 2}=12$, as described in the Supplemental Material of Ref. 20. We also changed $E_{1}=-3$ and $\delta=1$ to correspond approximately to

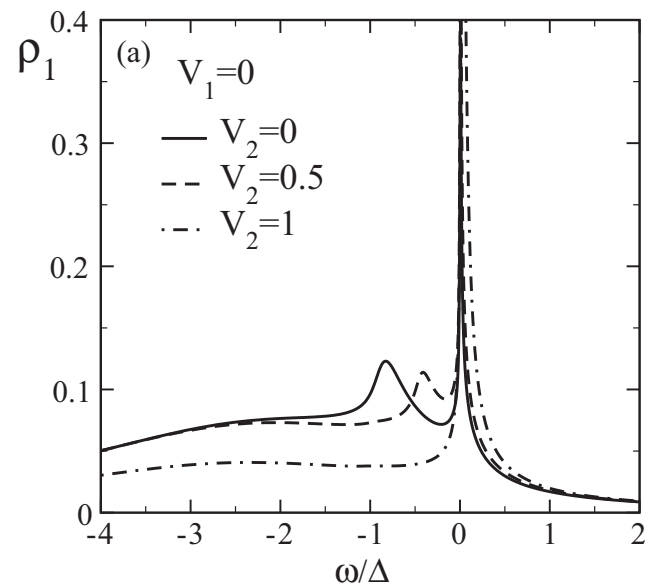

the experimental parameters. The evolution of $\rho_{1}(\omega)$ with $V_{S 2}$ is shown in Fig. 6(a). At equilibrium $\left(V_{S 2}=0\right)$, the spectral density of QD1 has the two peaks mentioned above. The inelastic peak can be understood as a mixture of the ground state for zero hopping with an excited state in which the electron at QD1 is displaced to QD2 and an an electron from $\mathrm{S} 2$ is displaced to D1. Both states are connected in second order in the lead-QDs' hopping. The excitation energy is $\delta$. As a consequence of this mixture, when an electron is destroyed in QD1, there is a finite probability of leaving an excited state with energy $\delta$. This leads to a peak at $-\delta$ in $\rho_{1}(\omega)$. When the chemical potential at $\mathrm{S} 2$ is increased, the excitation energy decreases and the peak displaces towards the Fermi energy. When this potential reaches $\delta$, the inelastic peak merges with the elastic one and this leads to a peak in $G_{1}\left(V_{1}\right)$ at $V_{1}=0$, even at temperatures above $T_{K}(\delta)$ for which the original elastic peak disappears. This agrees with the result presented in Fig. 4(d) of Ref. 20. We obtain a qualitative agreement with experiment, but the ratio of intensities is larger in our case. This might be due to uncertainties in the ratio $E_{1} / \Gamma_{i}$ or to fluctuations in $\delta$ introduced by decoherence effects. ${ }^{43}$

A similar reasoning as above can be followed for a symmetric voltage drop and brings an alternative explanation of the increase in intensity along the lines $e V_{1}= \pm e V_{2} \pm 2 \delta$ displayed in Fig. 3.

In Fig. 6(b) we show how $\rho_{2}(\omega)$ changes with $V_{S 2}$. In contrast to $\rho_{1}(\omega)$, much of the spectral weight lies above the Fermi energy. Therefore its magnitude is proportional to the amount of the singlet configuration without particles in the ground state, or in other words, to the degree of intermediate valence. We observe that $\rho_{2}(\omega)$ increases as $V_{S 2}$ approaches $\delta$.

In summary, we predict the values of the conductance through any of two capacitively coupled QDs as the voltage through any of them is varied. We believe that our results are important to stimulate further experimental research along the lines of recent pseudospin-resolved transport measurements. ${ }^{19,20}$ In particular, the presence of three peaks in $G_{1}\left(V_{1}\right)$ for $V_{2}=0$ (or two peaks in an STS configuration) with one of them at $\left(V_{1}\right)=0$ is characteristic of the $\mathrm{SU}(4) \rightarrow \mathrm{SU}(2)$ crossover. ${ }^{38}$ This has not been observed in experiment yet. ${ }^{19,20}$ However, giving the large experimental possibilities of tuning the parameters and the particular sensitivity of $T_{K}(\delta)$ with the

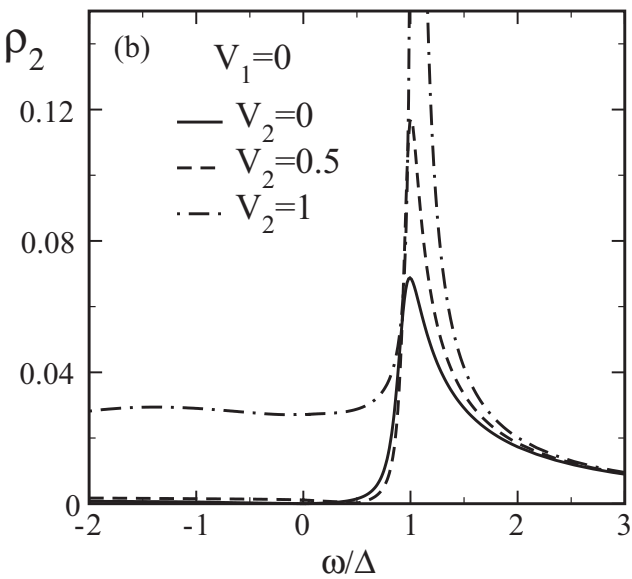

FIG. 6. Spectral density of (a) QD1 and (b) QD2 as a function of frequency for $E_{1}=-3, \delta=1$, and several bias voltages at source 2 . 
pseudo-Zeeman splitting $\delta$ we believe that it can be observed in the near future. An experimental study of the dependence of the lowest energy scale $T_{K}$ with the pseudo-Zeeman splitting $\delta$ would also contribute to our present understanding of the $\mathrm{SU}(4) \rightarrow \mathrm{SU}(2)$ crossover.
We thank A. J. Keller, G. Zaránd, S. Amasha, D. GoldhaberGordon, and A. M. Llois for useful discussions. This work was partially supported by PIP 112-200801-01821 and PIP-00273 of CONICET, and PICT 10-1060 and PICT 2011-1187 of the ANPCyT, Argentina.
${ }^{1}$ A. C. Hewson, The Kondo Problem to Heavy Fermions (Cambridge University Press, Cambridge, 1993).

${ }^{2}$ D. Goldhaber-Gordon, H. Shtrikman, D. Mahalu, D. AbuschMagder, U. Meirav, and M. A. Kastner, Nature (London) 391, 156 (1998).

${ }^{3}$ S. M. Cronenwet, T. H. Oosterkamp, and L. P. Kouwenhoven, Science 281, 540 (1998).

${ }^{4}$ D. Goldhaber-Gordon, J. Göres, M. A. Kastner, H. Shtrikman, D. Mahalu, and U. Meirav, Phys. Rev. Lett. 81, 5225 (1998).

${ }^{5}$ W. G. van der Wiel, S. de Franceschi, T. Fujisawa, J. M. Elzerman, S. Tarucha, and L. P. Kowenhoven, Science 289, 2105 (2000).

${ }^{6}$ L. Borda, G. Zaránd, W. Hofstetter, B. I. Halperin, and J. von Delft, Phys. Rev. Lett. 90, 026602 (2003).

${ }^{7}$ G. Zaránd, Philos. Mag. 86, 2043 (2006).

${ }^{8}$ P. Roura-Bas, L. Tosi, A. A. Aligia, and K. Hallberg, Phys. Rev. B 84, 073406 (2011).

${ }^{9}$ P. Jarillo-Herrero, J. Kong, H. S. J. van der Zant, C. Dekker, L. P. Kouwenhoven, and S. De Franceschi, Nature (London) 434, 484 (2005).

${ }^{10}$ J. S. Lim, M.-S. Choi, M. Y. Choi, R. López, and R. Aguado, Phys. Rev. B 74, 205119 (2006).

${ }^{11}$ F. B. Anders, D. E. Logan, M. R. Galpin, and G. Finkelstein, Phys. Rev. Lett. 100, 086809 (2008).

${ }^{12}$ S. Lipinski and D. Krychowski, Phys. Rev. B 81, 115327 (2010).

${ }^{13}$ C. A. Büsser, E. Vernek, P. Orellana, G. A. Lara, E. H. Kim, A. E. Feiguin, E. V. Anda, and G. B. Martins, Phys. Rev. B 83, 125404 (2011).

${ }^{14}$ L. Tosi, P. Roura-Bas, and A. A. Aligia, Physica B 407, 3259 (2012).

${ }^{15}$ K. Grove-Rasmussen, S. Grap, J. Paaske, K. Flensberg, S. Andergassen, V. Meden, H. I. Jorgensen, K. Muraki, and T. Fujisawa, Phys. Rev. Lett. 108, 176802 (2012).

${ }^{16}$ G. C. Tettamanzi, J. Verduijn, G. P. Lansbergen, M. Blaauboer, M. J. Calderón, R. Aguado, and S. Rogge, Phys. Rev. Lett. 108, 046803 (2012).

${ }^{17}$ P. Roura-Bas, L. Tosi, A. A. Aligia, and P. S. Cornaglia, Phys. Rev. B 86, 165106 (2012).

${ }^{18}$ E. Minamitani, N. Tsukahara, D. Matsunaka, Y. Kim, N. Takagi, and M. Kawai, Phys. Rev. Lett. 109, 086602 (2012).

${ }^{19}$ A. J. Keller, S. Amasha, I. Weymann, C. P. Moca, I. G. Rau, J. A. Katine, H. Shtrikman, G. Zaránd, and D. Goldhaber-Gordon, arXiv:1306.6326.

${ }^{20}$ S. Amasha, A. J. Keller, I. G. Rau, A. Carmi, J. A. Katine, H. Shtrikman, Y. Oreg, and D. Goldhaber-Gordon, Phys. Rev. Lett. 110, 046604 (2013).

${ }^{21}$ C. A. Büsser, A. E. Feiguin, and G. B. Martins, Phys. Rev. B 85, 241310(R) (2012).
${ }^{22}$ E. Vernek, C. A. Busser, E. V. Anda, A. E. Feiguin, and G. B. Martins, arXiv:1308.4746.

${ }^{23}$ Y. Nishikawa, A. C. Hewson, D. J. G. Crow, and J. Bauer, arXiv:1309.1715.

${ }^{24}$ M. Grobis, I. G. Rau, R. M. Potok, H. Shtrikman, and D. GoldhaberGordon, Phys. Rev. Lett. 100, 246601 (2008).

${ }^{25}$ A. Oguri, J. Phys. Soc. Jpn. 74, 110 (2005).

${ }^{26}$ A. A. Aligia, J. Phys.: Condens. Matter 24, 015306 (2012), references therein; Phys. Rev. Lett. 111, 089701 (2013); arXiv: 1310.8324.

${ }^{27}$ N. S. Wingreen and Y. Meir, Phys. Rev. B 49, 11040 (1994).

${ }^{28}$ M. H. Hettler, J. Kroha, and S. Hershfield, Phys. Rev. B 58, 5649 (1998).

${ }^{29}$ P. Roura-Bas, Phys. Rev. B 81, 155327 (2010).

${ }^{30}$ S. Florens, A. Freyn, N. Roch, W. Wernsdorfer, F. Balestro, P. Roura-Bas, and A. A. Aligia, J. Phys.: Condens. Matter 23, 243202 (2011), references therein.

${ }^{31}$ C. D. Batista and G. Ortiz, Adv. Phys. 53, 1 (2004).

${ }^{32}$ L. Tosi, P. Roura-Bas, and A. A. Aligia, J. Phys.: Condens. Matter 24, 365301 (2012)

${ }^{33}$ See Supplemental Material at http://link.aps.org/supplemental/ 10.1103/PhysRevB.88.235427 for details on the equations for the current and NCA formalism.

${ }^{34}$ U. Wilhelm, J. Schmid, J. Weis, and K. v. Klitzing, Physica E 14, 385 (2002).

${ }^{35}$ J. E. Moore and X.-G. Wen, Phys. Rev. Lett. 85, 1722 (2000).

${ }^{36}$ L. Vaugier, A. A. Aligia, and A. M. Lobos, Phys. Rev. B 76, 165112 (2007).

${ }^{37}$ A. Freyn and S. Florens, Phys. Rev. Lett. 107, 017201 (2011).

${ }^{38} \mathrm{~A}$ differential conductance with three peaks has been also observed in transport through $\mathrm{C}_{60}$ quantum dots in which triplet states are important (Refs. 30 and 39) and explained by NCA calculations (Refs. 30, 40, and 41). However, the dependence of gate voltage of the position of the peaks is quite different (Ref. 30).

${ }^{39}$ N. Roch, S. Florens, V. Bouchiat, W. Wernsdorfer, and F. Balestro, Nature (London) 453, 633 (2008).

${ }^{40}$ P. Roura-Bas and A. A. Aligia, Phys. Rev. B 80, 035308 (2009).

${ }^{41}$ P. Roura-Bas and A. A. Aligia, J. Phys.: Condens. Matter 22, 025602 (2010).

${ }^{42} \mathrm{An}$ example of the limitations of NRG to detect finite-energy features is the plateau at intermediate temperatures observed in transport through $\mathrm{C}_{60}$ molecules for gate voltages for which triplet states are important (Refs. 30 and 39), which was missed in early NRG studies but captured by the NCA (Refs. 40 and 41). More recent NRG calculations using tricks to improve the resolution (Ref. 37) have confirmed this plateau (Ref. 30).

${ }^{43}$ A. J. Keller (private communication). 